\title{
Neurostimulation and Therapeutic Advance: Iterative or Principled?
}

\author{
Denis Larrivee ${ }^{1,2 *}$ \\ ${ }^{1}$ Loyola University Chicago, USA \\ ${ }^{2}$ Mind and Brain Institute, University of Navarra Medical School, Spain
}

*Corresponding author: Denis Larrivee, Mind and Brain Institute, University of Navarra Medical School, Spain.
Received Date: July 21, 2020

Published Date: August 05, 2020

\begin{abstract}
Summary
Although electrical stimulation has been used intermittently for centuries to treat neural dysfunction, recent decades have seen a renaissance, with therapeutic applications ranging from motor disorders like dystonia and Parkinson's to urological dysfunction and even psychiatric diseases. Fueling growth is an improved capacity for targeted spatial and temporal intervention over that of pharmacological methods. On the other hand, despite an improving understanding of its mechanisms of action, the complexity of higher order neural mechanisms and conceptual conflicts over the structuring of global brain activity continue to overshadow development, making advances on the basis of principle the exception rather than the norm.
\end{abstract}

Keywords: Neurostimulation; Electrotherapy; Radical predictive processing; Brain oscillations; Global brain states; Epilepsy; Neural dysfunction; Parkinson's disease

\section{Introduction}

For centuries, electrical stimulation has been employed for treating neural disorders [1]. As early as the Egyptian period, medical records cite the use of electric eel shock therapy for pain while in the early scientific era Johann Kruger, a professor of medicine and philosophy in Halle, Germany, advocated electrotherapy for paralyzed limbs, a practice his pupil Kratzenstein later mastered. Kratzenstein's treatments, for instance, claimed a complete restoration of movement, with especially dramatic recoveries in musicians. Late in the 1700's British clerics John Wesley and Richard Lovett reported successfully treating hysterical disorders, as did Benjamin Franklin in the Americas - enthusiastically recorded by one patient.

'I received four shocks morning and evening; they were what they call 200 strokes of the wheel, ..... the symptoms gradually de creased, till at length they intirely [sic] left me... I now enjoy such a state of health, as I would have given all the world for, this time two years, if it had been in my power, and I have great reason to hope it will continue.'

Interest in electrotherapy waned in the late 1800s and early 1900s as psychotherapies came to acquire greater prestige for treating psychiatric symptoms. In recent decades, however, interest in neurostimulation for therapy has again reignited, beginning chiefly with applications for motor disorders [2]. For patients with Parkinson's Disease (PD) high-frequency stimulation of the subthalamic nucleus or globus pallidus was found to produce clinical effects similar to surgical ablation, yet was also reversible, unlike surgery [3]. Studies into treatment efficacy revealed that low frequency stimulation (4-8Hz, occasionally $25 \mathrm{~Hz}$ ) of the pallidum 
elicited tremors whereas high frequency stimulation $(25-100 \mathrm{~Hz})$ abolished them. Although the invasive introduction of electrodes initially made these surgeries less popular than pharmacological therapies using levodopa, the emergence of complications from long-term levodopa treatment - including motor fluctuations and dyskinesias (LID) - led again to widespread use of neurostimulation.

Due to the successes in symptomatic and longterm therapeutic relief, neurostimulatory and neuromodulatory protocols have also seen rapid growth in domains other than the motor diseases. Fueling growth, moreover, are the intrinsic advantages of neurostimulation over the more commonly used pharmaceutical agents. Unlike pharmacological approaches, which are difficult to target to specific brain regions and whose temporal distribution is usually prolonged, neurostimulation is inherently better suited to address the discrete spatial and temporal variation that characterizes individual dysfunctions. Despite its requirement for invasively embedding electrodes, the precision of deep brain stimulation, for instance, has warranted its use for treating a number of refractory neurological, movement and psychiatric related, disorders. In movement disorders, for example, DBS has been shown to be therapeutic for tremors, dystonia, and dyskinesia, yielding a 50\% improvement in PD on the Unified Parkinson's Disease Rating Scale. Among the psychiatric diseases DBS has shown promise for treatment of obsessive-compulsive disorder and Gilles de la Tourette syndrome, with a limited impact on major depressive syndrome [2].

The need for surgical intervention with its risk of secondary complications, moreover, has itself been the stimulus for developing an additional, equally broad group of non-invasive applications. These applications have tended generally toward neurological disorders that display symptoms distinguished from those treated by DBS and have also seen use in diagnostic applications [4,5]. Among these are such established non-invasive technologies as transcranial magnetic stimulation (TMS), transcranial direct current stimulation (tDCS), and transcranial alternating current stimulation (tACS). Repetitive TMS (rTMS), for instance, has been shown to promote the recovery of limb motor function in patients with stroke. Diagnostically, TMS can also be a surrogate marker of recovery that is both sensitive and quantitative for cranial vascular disorders. TMS has proven to be useful for examining cortical and corticospinal physiology and to enable the understanding of motor dysfunction, for example. When physiologic data are correlated with clinical function, they can assist diagnosis of underlying motor dysfunction. Operant conditioning with TMS, for instance, has been used both to assess the weakness of corticospinal connections and to promote targeted neuroplastic change [6].

\section{An Improving Understanding of Brain Communica- tion}

While the mechanisms elicited by neurostimulation are largely unknown, an improving understanding of how brain activity is constructed is likely to facilitate the development of the technology. It has notably been proposed that because electrical activity is fundamental to brain communication, neurostimulation somehow affects the electrical patterning that enables communication. The understanding of how patterning is structured, however, is both evolving and vigorously debated, with the result that mechanisms of neurostimulation have themselves been poorly understood. Information coding, for example, has dominated scientific discussion for decades, yet how coding occurs is unknown. One of the pre-eminent theories about information processing, proposed by Hubel and Wiesel in the 1970s on the basis of their discovery of edge and motion detector cells [7], is the progressive hierarchical abstraction of information by higher order neurons. At the apex of this neuronal hierarchy, cardinal neurons were posited to encapsulate highly complex representations of the external world, a conception that effectively limited the number of representations to a few principal neurons. In the 1990's Singer proposed a new thesis premised on the combinatorial properties of brain oscillations, which were intrinsic to neural operation [8]. These properties included the combining of brain oscillators by synchronization and their separation by desynchronization, allowing for a virtually unlimited number of representations. The new proposal overcame the theoretical constraints of the Hubel and Wiesel model and gave neural communication virtually unlimited freedom of expression. Further developments revealed that the temporal patterning of neuronal discharges by means of delay coupled oscillator networks enabled the formation of select relations between distributed assemblies of neurons [9]. These developments suggested an even broader potential for representational options through the introduction of variations in temporal sequencing. Among the many examples of the latter are the dynamic formation of functional networks, selective routing of activity in densely interconnected networks, attention-dependent selection of sensory signals, fast and context-dependent binding of responses for joint processing in pattern recognition, and the formation of associations by learning [10].

On the other hand, despite the improved understanding of brain activity, the complexity of the brain's functional organization continues to pose significant hurdles to targeted neurostimulation. The complexity of this influence is likely to relate to several properties known to characterize neural oscillations that are intrinsic to the principle oscillatory patterns seen in normal brain operation $[11,12]$. Disease states are known to alter these properties, modifying oscillatory behaviour and affecting how neurostimulation can in turn reverse the effects of the disease. The intrinsic ability of oscillators to combine through synchronization, for example, can be evoked via a number of mechanisms such as phase and amplitude coupling [8] and various forms of cross frequency coupling that configure the conditions for how oscillators align and then resonate in unison. Due to their ubiquity throughout the brain they are capable of coordinating with global (proposed 
to occur through slower oscillations like theta and delta waves), and/or regional (thought to involve gamma waves) activities [12]. The effect of cross frequency coupling in epilepsy, for example, is known to underlie the distribution of epileptogenesis throughout the cortex [13]. Resonating in unison has the important effect of enabling information transfer and so of regulating communication between brain domains [14]. Interruptions in normal synchronous activity, therefore, can prevent communication.

Oscillators must also disengage through desynchronization, where their frequencies are no longer aligned, to generate new combinations with functionally different outcomes. For example, both beta and mu basal ganglia rhythms show event related desynchronization prior to movement in the basal ganglia, with sustained suppression during movement execution [15]. Desynchronization requires a discrete segregation of oscillator pairs to avoid functional overlap; that is, a qualitative bifurcation of the two that is fundamentally a non-linear and dynamical event, for which several mechanisms are proposed [16], like phase resetting through pulsing, or noise induced effects that entrain localized rhythms [17]. Phase shifting, or curve resetting, is a common mechanism for oscillatory control, one already implicated in Wilson Cowan excitatory inhibitory models. By means of spike timing dependent plasticity oscillatory phases can be advanced or delayed affecting when and how separation may occur [11], a feature that must be accounted for in adjusting aberrant combinations.

Synchronization and desynchronization notably help the brain to maintain stability as well as to achieve the flexibility needed for functional variance. For a noisy information processing system like the brain, stability is a critical feature; internal states must be reasonably stable - otherwise the whole system would vascillate, leading to erratic behavior. Flexibility through desynchronization, on the other hand, provides for the option to vary oscillator combinations, and so yield variation in the routing of information. Due to the need to transition from synchronized to desynchronized state, thus, the level of stability must also change as combinations are formed and then disengaged, affecting how trajectory routing occurs and which oscillator combinations are made.'

Transitioning (and so changes in the degree of stability) of this sort is theoretically modeled by means of elements termed 'attractors', that is, points to which an activation variable of a system, e.g., firing rates, always returns after small perturbations, limiting the effect of perturbation introduced through neurostimulation [16]. Such stable points are often mathematically described as functions that depend on the prior state of the system. As the system departs from its most stable configuration due to a perturbation, the attractor exerts an attractive force that is proportional to the size of the deviation induced by the perturbation. Points along the trajectory, those initially induced by the perturbation and then those induced by the attraction of the attractor, represent, therefore, points of lesser stability within the attractors of a system. For a simple, general linear system of the form

$$
\delta \mathrm{u} / \delta \mathrm{t}=-\mathrm{u}+\mathrm{h}
$$

where $u$ represents an activation variable, e.g., firing rate, and $\mathrm{h}$ is the resting level the relationship between time and distance to the attractor can be described by the exponential function:

$$
u(t)=e-\beta t(u(0)-h)+h
$$

where $u(0)$ is the initial state at $t=0[18]$

The time it takes for the system to reduce the perturbation by half of the initial distance from the attractor $(r=0.5)$, can be shown to depend upon the parameter $\beta$, and is conventionally taken as 0.368 , i.e., $\mathrm{e}^{-1}$. On the other hand a number of more complex attractors have been shown to exist, with more complex adjustments needed during neurostimulation.

Crucially, attractors are framed as components of a global operation; meaning the brain and, ultimately, the whole organism, the latter defining the sorts of mechanisms required for the 'good' of the whole [19]. This means that the resident oscillator field is not unimodal, but rather represents a broad distribution of oscillator attractors elicited by the network connectivity and determined by the physical parameters that give rise to them, such as impedance resonance and varied anatomical configurations [11]. Neurostimulation may thus drive synchrony with a subset of oscillators but may do so only if the relative coupling between the source of neurostimulation and the neural oscillators are energetically preferred to other combinations, where local instabilities might otherwise disengage them [18]. Accordingly, in the targeting of particular oscillators, neurostimulation must take into account large oscillator fields, which possess an overall global architecture within which oscillatory interactions are structured.

Selection within such fields thus entails circumstances of competition between competing attractor elements. A slight difference in activation can translate into a difference that will shift the interaction to the other attractor. Hence, presenting a stimulus modifies attractor stability, moving the system into a bistable regime and overcoming random fluctuations to determine which attractor is selected. Once relaxation has occurred the system dynamics stabilize the system state against further perturbations; that is, a stable decision between the two competing attractors is made. The presence of a large attractor field means, however, that multiple decisions are likely to be made, a factor that has prompted the development of Dynamic Field Theory (DFT) [20]. The theory, accordingly, has attempted to elaborate modeling provisions for detecting selection decisions and the tracking of time varying input due to instabilities in the neural dynamics.

For oscillators in the dynamic environment of the dynamic environment of the brain, moreover, points of greatest stability occur during synchronization when phases are constant with respect to each other, a circumstance termed phase locking. However, rigid or full phase locking is rarely if ever achieved [21,22], a natural physical feature permitting the separation of pair members to acquire 
the corresponding potential to form new combinations. Because perfect synchrony is not strictly attainable the effect of increasing coupling strength is to increase synchronization residency within a preferred overlap range where oscillator frequency differences are minimal, mathematically described by the sine of the phase angle difference between oscillators. As a result the oscillators continue to experience frequency modulation throughout the cycle, which is manifest in the continual change in their precession rates. While it has been proposed that frequency modulation (rather than synchronization per se) provides for information transfer, neurostimulation can be expected to affect modulation and to impact spectral power, influencing factors like the extent of phase alignment in the population [17], frequency modulation due to the phase dependency of the coupling constant [21], intermittency of alignment [22,23], and oscillator disruption that may be occasioned by excessive coupling strength [22]. Indeed, the need for oscillators to recombine requires that synchronization be of only modest strength, and thus susceptible to neurostimulatory effects. This requirement has led to the current model for oscillator pairing, the Theory of Weakly Coupled Oscillators [11,21], mathematically described by the Adler equation, which includes terms for repulsion, termed detuning, and for coupling, both of which vary as a function of the frequency difference between pair members. (Weak here means that interactions lead to phase adjustments without strong perturbations of the oscillatory generative mechanisms.)

For neurostimulation precise temporal and spatial resolution constitutes an ideal objective, with limited interrogation intervention. However, due to the dynamic nature of the oscillator field the effects of carefully circumscribed stimuli can be shortlived [24]. Critically, as noted, such impact is highly dependent on the state of the field.

Because of the field's dynamic character, many perturbation paradigms have opted to either use a very strong pulse, essentially resetting and disrupting the activity of the target network, or to use a continuous or repetitive pulse stimulation in order to establish and maintain a desired effect. One study [25] that entrained a local neuron population with a $40 \mathrm{~Hz}$ train of pulses showed that the neural population response as well as the animal behavior depended on the phase at which the stimulus arrived at the population. While these studies illustrate effects introduced by stimulation, a number of concerns become apparent with these regimes due to the dynamic nature of brain activity. Stimulation can, for instance suppress any ongoing local processing and so interfere and contaminate the relevant neural signals. The introduction of stimuli, moreover, clearly affects the stability of oscillator associations, modulating information transfer in unexpected ways.

\section{Conceptual Conflicts over Global Brain Dynamics}

Besides the mechanistic difficulties that are due to the dynamic nature of brain state activity, philosophical impediments on the nature of the brain's functional organization also overshadow growth, rendering development of the technology on a scientifically principled basis a difficult to achieve goal. While the elucidation of mechanistic features of brain operation is ongoing, how features like oscillatory patterning are functionally organized remains elusive. One of the fundamental obstacles to deciphering brain operation is that of competing visions of the brain's relation to the body, which have become acute with attempts to understand how brain function is unified. This conceptual uncertainty about the brain overshadows therapeutic advance. A traditional understanding, inherited from Ramon de Cajal, posits that the body is wholly subordinate to the brain's regulation, a position that has become enshrined, for instance, in the clinical praxis and legal polity of death determinations. By this understanding all computational and regulatory processes originate within the brain, typically in the form of a priori neural representations through which the body is modulated as the brain directs. A widely endorsed model, for example, the radical predictive processing model [26], proposes that bodily movements are computed as neural priors, which evoke corresponding action sequences that are monitored for their congruency with the internal prior. Such models reserve to the brain all significant computational determinations needed to guide action trajectories. Opposing this brain-centered view, another conception sees the relation between body and brain as mutually and reciprocally interactive, with the body's 'states' significantly and continually modulating brain activity [26]. It is known, for example, that during development ongoing afferent activity shapes the cortical synaptic architecture, a process that is also induced experientially throughout the life of the organism. The effect of such conceptual differences for neurostimulation therapy lies in the uncertainty surrounding which brain properties are actually being modulated; that is, how the electrical features in fact relate to the behavioral or clinical manifestations that are impaired. How global brain activity may be affected by input from the body influences functional organization and so can be expected to be a crucial element in applying neurostimulation.

The presence of a global order is notably apparent in dysfunctions like epilepsy. A working definition of this disorder states that it is 'characterized by a persistent predisposition to generate at least one epileptic seizure and by the neurobiological, cognitive, psychological and social consequences of this condition' [27]. Seizures can cause various sequelae that entail brief changes in perception and behavior, mild convulsions, and temporary loss of consciousness, that appear to relate to seizure origin and the degree of their intensity. This is to say that epileptic events can entail broad activity changes across the brain. Among the often profound changes occurring during epileptic episodes, for instance, are altered states of consciousness, which are likely to involve major networks outside the region of seizure origin. Consistent with such observations, functional connectivity is impaired in large scale brain networks that extend both bilaterally and via subcortical structures [28]. Recurring seizures, therefore, implicate the presence of large-scale interactions as major pathogenic factors contributing to symptom severity. Findings from patients resistant 
to treatment with anti-epileptic drugs, in fact, strongly suggest this. Because the probability of resistant patients to achieve complete remission with new antiepileptic drugs is less than $10 \%$, surgical intervention is often considered the best option for treating intractable epilepsy [29]; however, after temporal lobe and/or localized neocortical resections only $29-65 \%$ of the patients are free of seizures. This relatively low success rate has prompted a number of studies on the mutual, excitatory and inhibitory interrelations of brain structures participating in epileptogenesis, which have converged on a proposal of epileptic systems that develop in the brains of these patients. The existence of such complex epileptic systems could explain the intractability of epilepsy and the lack of success of resective surgery. In temporal lobe epilepsy, for instance, synchronization of activity between the temporal lobe and thalamus has been correlated with alterations of consciousness, a finding among others that has suggested that consciousness resides only within the confines of the brain. A prominent model of consciousness, for instance, postulates that consciousness entails a continually shifting workspace within the brain [30]. Yet it is also known that afferent input from the body plays a crucial role in activating and sustaining consciousness. How or which aspects of consciousness may be modulated by neurostimulation during epileptic AOC therefore remain uncertain, with the result that therapeutic efficacy is itself compromised.

Another key, higher order structure likely to be affected by bodily input, the default mode network (DMN), has also been shown to be impaired by epilepsy [31]. The DMN is a major resting network that enables transitioning between task negative and task positive states, with functional communication occurring through the basal ganglia. Significantly, several experimental and clinical studies show that the putamen and other BG nuclei are likely to modulate epileptic seizures, with changes in functional connectivity between the DMN and ganglia apparently the source of this modulation. Significantly, task positive motor activity of the DMN is intimately linked to 3D postural representations of the whole body in the parietal cortex. There is increasing evidence that discrete motions are framed in the context of the whole body's posture and that the dynamic whole is continually updated as an ongoing loop with the body's motor activity. How this may be altered in epilepsy is unknown.

Attempts to resolve these uncertainties have shown just how deep the philosophical divide may go, pitting conceptions about how organisms cohesively operate in the world against traditional research strategies premised on the scientific method. Ashbury, notably, famously argued that every good regulator is a model of its system [32]. Extended to the brain - as he also intended - this means that in every neural function its global regulation can be perceived. In simple organisms like C. elegans global regulation is known to be mediated by the organism's generation of a a global action state from neurons throughout its body, to which the activity of individual motor commands is subordinated and synchronized
[33]. In advanced organisms like ourselves, we know relatively little of how global oscillatory patterns emerge, but if findings from primitive organisms like $\mathrm{C}$ elegans are generalizable it is likely that the body will be fundamentally involved in generating this global activity state. Modulating patterns like these by neurostimulation will almost certainly have, to greater or lesser degrees, systemic consequences that apply to the individual as a whole.

\section{Conclusion}

While therapeutic applications for neurostimulation are expanding, advances on the basis of theory continue to encounter significant hurdles. Beyond the dynamical character of brain activity, competing philosophical models over the body's contribution to structuring global functions have yet to resolve. In the absence of a resolution to these philosophical concerns, understanding how body and brain are unified is likely to remain a crucial obstacle to the advance of neurostimulation therapy on the basis of theory. This is to say that iterative approaches will continue to remain effective, but not principled, strategies for the development of new therapeutic options.

\section{Acknowledgement}

None.

\section{Conflict of Interest}

No conflict of interest.

\section{References}

1. Heidland A, Fazeli G, Klassen A, Sebekova K, Hennemann H, et al. (2013) Neuromuscular electrostimulation techniques: historical aspects and current possibilities in treatment of pain and muscle waisting. Clin Nephrol Suppl 1: S12-23.

2. Udupa K, Chen R (2015) The mechanisms of action of deep brain stimulation and ideas for the future development. Progress in Neurobiol 133: 27-49.

3. Benabid AL, Pollak P, Louveau A, Henry S, de Rougemont J (1987) Combined (thalamotomy and stimulation) stereotactic surgery of the VIM thalamic nu- cleus for bilateral Parkinson disease. Appl. Neurophysiol 50: 344-346.

4. MarCortes, Randie M Black-Schaffer, Dylan JEdwards (2012) Transcranial Magnetic Stimulation as an Investigative Tool for Motor Dysfunction and Recovery in Stroke: An Overview for Neurorehabilitation Clinicians. Neuromodulation 15: 316-325.

5. Maria Ioanna Stefanou, Debora Desideri, Paolo Belardinelli, Christoph Zrenner, Ulf Ziemann (2018) Phase Synchronicity of a-Rhythm Determines Efficacy of Interhemispheric Communication Between Human Motor Cortices Journal of Neuroscience 38(49): 10525-10534.

6. Thompson AK, Cote RH, Sniffen JM, Brangaccio JA (2018) Operant conditioning of the tibialis anterior motor evoked potential in people with and without chronic incomplete spinal cord injury J Neurophysiol 120: $2745-2760$

7. Wurtz RH (2009) Recounting the impact of Hubel and Wiesel. J Physiol 587(12): 2817-2823.

8. Singer W (1999) Neuronal synchrony: a versatile code for the definition of relations? Neuron 24: 49-65.

9. Singer W (2018) Neuronal oscillations: unavoidable and useful? European J Neurosci 48: 2389-2398. 
10. Havenith MN, Yu S, Biederlack J, Nan-Hui Chen, Wolf Singer, et al. (2011) Synchrony makes neurons fire in sequence, and stimulus properties determine who is ahead. J Neurosci 31: 8570-8584.

11. Wang XJ (2010) Neurophysiological and computational principles of cortical rhythms in cognition. Physiological Review 90: 1195-1268.

12. Buzsaki G (2006) Rhythms of the Brain. New York: Oxford University Press.

13. Sheybani L, Pieter van Mierlo, Gwénaël Birot, Christoph M. Michel, Charles Quairiaux (2019) Large-scale 3-5 hz oscillation constrains the expression of neocortical fast ripples in a mouse model of mesial tem poral lobe epileps. eNeuro 6(1): e0494-e0418.

14. Lowet E, Mark J Roberts, Pietro Bonizzi, Joël Kare, Peter De Weerd (2015) Quantifying neural oscillatory synchronization: a comparison between spectral coherence and phaselocking value approaches. PLoS One 11: e0146443.

15. Doesburg SM, Jessica J Green, John J McDonald, Lawrence M Ward (2009) From local inhibition to long-range integration: a functional dissociation of alpha-band synchronization across cortical scales in visuospatial attention. Brain Research 1303: 97-110.

16. Reimann $\mathrm{H}$ et al (2015) The dynamics of neural activation variables Paladyn J Behav Robot 6:57-70.

17. Canavier CC (2015) Phase resetting as a tool of information transmission Current Opin. Neurobiol. 31: 206-213.

18. Schöner G, Dineva E (2007) Dynamic instabilities as mechanisms for emergence. Develop Sci 10: 69-74

19. Friston K (2013) Free energy and global dynamics. In: Principles of Brain Dynamics: Global State Interactions. In: Rabinovich MI, Friston KJ, Varona P (Eds.), Cambridge, MA: MIT Press, USA.

20. Schoner G (2009) Development as change of system dynamics: stability, instability, and emergence. In Toward a Unified Theory of Development. Spencer J, Thomas MSC, McClelland JL (eds). Oxford: Oxford University Press.

21. Lowet et al. (2017) A quantitative theory of gamma synchronization in macaque V1. eLife 6: e26642.
22. Larrivee D (2018) Global dynamics and local synchrony: therapeutic prospects for implant learning devices. Internat Joint Conf Neural Networks 1-6.

23. Ahn S, Rubchinsky LL (2013) Short desynchronization episodes prevail in synchronous dynamics of human brain rhythms. Chaos 23: 1-8

24. Lisitsyn D, Ernst UA (2019) Causally investigating cortical dynamics and signal processing by targeting natural system attractors with precisely timed (electrical) stimulation. Frontiers Comput Neurosci 13(7).

25. Cardin JA (2009) Driving fast-spiking cells induces gamma rhythm and controls sensory responses. Nature 459: 663-667.

26. Allen M, Friston KJ (2018) From cognitivism to autopoiesis: towards a computational framework for the embodied mind. Synthese 195 2459-2482.

27. Curia G, C. Lucchi, J Vinet, F Gualtieri, C Marinelli, et al. (2014) Pathophysiogenesis of mesial temporal lobe epilepsy: is prevention of damage antiepileptogenic? Current Medicinal Chemistry 21(6): 663688.

28. Larrivee D (2020) Brain network and homeostatic influences in epileptogenic spreading. EC Nutrition 15(3): 01-09.

29. Benbadis SR, Eric Geller, Philippe Ryvlin, Steven Schachter, James Wheless, et al. (2018) Putting it all together: Options for intractable epilepsy. An updated algorithm on the use of epilepsy surgery and neurostimulation Epilepsy Behav 88: 33-38.

30. Dehaene S, Changeux JP (2001) A neuronal model of a global workspace in effortful cognitive tasks. Proc Natl Acad Sci 95(24): 14529-14534.

31. Rektor I, Jan Tomč́́k, Michal Mikl, Radek Mareček, Milan Brázdil, et al. (2013) Association between the basal ganglia and large-scale brain networks in epilepsy. Brain Top 26: 355- 362.

32. Eykhoff P (1995) Every good regulator of a system must be a model of that system. Moedling, Identif, Control 15(3): 135-139.

33. Kato S, Harris S Kaplan, Tina Schrödel, Susanne Skora, Theodore H Lindsay (2015) Global brain dynamics embed the motor command sequence of caenorhabditis elegans. Cell 163: 656-669. 